Modelling the spatio-temporal modulation response of ganglion cells with difference-of-Gaussians receptive fields : Relation to photoreceptor response kinetics

Donner, K.

Cambridge University Press

1996

Visual Neuroscience. 1996. 13: 173-186

http://hdl.handle.net/1975/952

Downloaded from Helda, University of Helsinki institutional repository.

This is an electronic reprint of the original article.

This reprint may differ from the original in pagination and typographic detail.

Please cite the original version. 


\title{
Modelling the spatio-temporal modulation response of ganglion cells with difference-of-Gaussians receptive fields: Relation to photoreceptor response kinetics
}

\author{
KRISTIAN DONNER ${ }^{1}$ AND SIMO HEMILÄ ${ }^{2}$ \\ 'Department of Biosciences and Department of Ecology and Systematics, P.O. Box 17, \\ FIN-00014 University of Helsinki, Finland \\ ${ }^{2}$ Laboratory of Physics, Helsinki University of Technology, FIN-02150 Espoo, Finland
}

(ReCEIVEd August 5, 1994; ACCEPTED June 20, 1995)

\begin{abstract}
Difference-of-Gaussians (DOG) models for the receptive fields of retinal ganglion cells accurately predict linear responses to both periodic stimuli (typically moving sinusoidal gratings) and aperiodic stimuli (typically circular fields presented as square-wave pulses). While the relation of spatial organization to retinal anatomy has received considerable attention, temporal characteristics have been only loosely connected to retinal physiology. Here we integrate realistic photoreceptor response waveforms into the DOG model to clarify how far a single set of physiological parameters predict temporal aspects of linear responses to both periodic and aperiodic stimuli. Traditional filter-cascade models provide a useful firstorder approximation of the single-photon response in photoreceptors. The absolute time scale of these, plus a time for retinal transmission, here construed as a fixed delay, are obtained from flash/step data. Using these values, we find that the DOG model predicts the main features of both the amplitude and phase response of linear cat ganglion cells to sinusoidal flicker. Where the simplest model formulation fails, it serves to reveal additional mechanisms. Unforeseen facts are the attenuation of low temporal frequencies even in pure center-type responses, and the phase advance of the response relative to the stimulus at low frequencies. Neither can be explained by any experimentally documented cone response waveform, but both would be explained by signal differentiation, e.g. in the retinal transmission pathway, as demonstrated at least in turtle retina.
\end{abstract}

Keywords: Retina, Contrast sensitivity, Flicker, Photoreceptors

\section{Introduction}

Optical factors aside, spatio-temporal resolution and contrast sensitivity are fundamentally limited by the sampling and filtering properties of retinal neurons. The approach towards a full physiological understanding of these limitations has been retarded partly by the conceptual gap between functionally and mechanistically oriented studies. Following de Lange's (1952, 1958) pioneering work, the study of visual function has been guided by a systems approach, with a parallel tradition of retinal research typically using sinusoidal stimulation patterns and frequency-domain analysis (Enroth-Cugell \& Robson, 1966; Baron \& Boynton, 1975; Derrington \& Lennie, 1982; Linsenmeier et al., 1982; Enroth-Cugell et al., 1983; Frishman et al., 1987; Victor, 1987; Chen \& Freeman, 1989; Troy et al., 1993).

Reprint requests to: Kristian Donner, Department of Biosciences, P.O. Box 17, FIN-00014 University of Helsinki, Finland.
Physiological mechanism, on the other hand, is often more transparent to experiments using flash-step, spot-annulus, i.e. "aperiodic" stimulation paradigms (ganglion cell receptive fields: Barlow, 1953; Kuffler, 1953; Rodieck \& Stone, 1965a,b; Creutzfeldt et al., 1970; Enroth-Cugell \& Shapley, 1973; Enroth-Cugell \& Lennie, 1975; Peichl \& Wässle, 1979; photoreceptor response waveform: Fuortes \& Hodgkin, 1964; Baylor \& Fuortes, 1970; Baylor et al., 1974, 1979, 1984; Tamura et al., 1989; Schnapf et al., 1990; Kraft et al., 1993). Graham and Hood (1992) have stressed the importance of integrating "periodic" and "aperiodic" research traditions in psychophysics. In retinal research, this objective is closely related to reaching a real physiological understanding of the system properties.

Difference-of-Gaussians (DOG) models for ganglion-cell receptive fields (GC RFs) as introduced by Rodieck and Stone $(1965 a, b)$ and Rodieck (1965) and elaborated over 30 years (Enroth-Cugell \& Robson, 1966; Grüsser \& Grüsser-Cornehls, 1973; Hochstein \& Shapley, 1976a,b; Derrington \& Lennie, 1982; Enroth-Cugell et al., 1983; Dawis et al., 1984; Troy et al., 
1993) go far towards true anatomical/physiological understanding of linear spatial processing. On one hand, they effectively predict responses to a wide variety of both periodic and aperiodic stimuli. On the other hand, the basic concepts - antagonism of spatially overlapping mechanisms and the Gaussian sensitivity distribution of at least the RF center - can be fairly convincingly identified with specific anatomical and physiological substrates (e.g. Creutzfeldt et al., 1970; Wässle \& Creutzfeldt, 1973; Enroth-Cugell \& Lennie, 1975; Wässle et al., 1981; Peichl \& Wässle, 1983). By contrast, the central temporal concepts (the modulation transfer function, phase shifts of center and surround) remain physiologically complex. Although the physiology of the primary temporal filters in the retina, the rods and cones, has been extensively characterized, realistic photoreceptor response waveforms have not been explicitly integrated into the DOG model.

\section{Approach}

At one level, neural modelling should always strive to associate basic features with specified anatomical and physiological substrates. Even where oversimplified, models with an appropriate level of physiological specificity serve to factor out (or, conversely, falsify) the "obvious" and point to additional mechanisms. Our idea is to see how far a minimum of rather simplistic assumptions will go towards explaining the temporal (strictly speaking, spatio-temporal) response of linearly operating GCs. Importantly, insisting on absolute values for physiological parameters enables a strict comparison between aperiodic and periodic results.

The linearity assumption is of course a restrictive one and some would feel that by making this assumption we exclude most that is really interesting. However, our present purpose requires simplification as much as possible, physiologically as well as mathematically. The results still have considerable generality. Photoreceptors respond linearly to low-contrast stimuli. Synaptic transfer is largely linear for small signals. "Nonlinear" GC classes have linear response components, which can be isolated by appropriate experimental procedures (Hochstein \& Shapley, 1976b; Frishman \& Linsenmeier, 1982; Troy et al., 1993).

General relations are derived by conventional linear systems theory. The physiological parameters introduced are associated with four basic entities: (1) the sensitivity profile of the RF center (RFC); (2) the sensitivity profile of the antagonistic RF surround (RFS); (3) the waveform of the single-photon response in photoreceptor cells; and (4) the delay for signal transmission from photoreceptors to GCs, differing in the center and the surround pathways. It should be realized that center/surround antagonism is an equally essential feature of temporal as of spatial organization.

\section{Physiological elements}

\section{The spatial sensitivity distribution of the receptive-field center}

The RFC sensitivity profile is a spatial weighting function that describes the relative strength of synaptic coupling between the GC and a photoreceptor at a certain retinal point. In the DOG model, the distribution is Gaussian and in the simplest case radially symmetric. Relative sensitivity $z(r)$ (a dimensionless quan- tity) is then a monotonically decreasing function of distance $r$ from the RF midpoint, placed in the origin:

$$
z(r)=\exp \left(-r^{2} / r_{0}^{2}\right)
$$

The absolute sensitivity at any point is $z(r)$ multiplied by the peak sensitivity $Z_{0} . r_{0}$ is the effective radius of the RFC. The effective area is defined by

$$
A_{i}=\pi r_{0}^{2}=\int_{-\infty}^{\infty} \int_{-\infty}^{\infty} z(x, y) \mathrm{d} x \mathrm{~d} y
$$

If the Gaussian is substituted by a "top hat" distribution with the same peak sensitivity and the same integrated sensitivity, $r_{0}$ and $A_{i}$ are the radius and area of the latter.

The Gaussian is a priori a plausible idealization in view of the cascaded distributions of radially decreasing dendritic densities in the neuronal transmission chain from receptor to ganglion cell. Experimentally, it is consistent with RFC profiles mapped by small spots and slits of light, or by area-sensitivity functions (Rodieck \& Stone, 1965a, $b$; Creutzfeldt et al., 1970; Wässle \& Creutzfeldt, 1973; Peichl \& Wässle, 1979).

\section{The spatial sensitivity distribution of the antagonistic surround}

In the DOG model, the sensitivity distribution of the RFS is also Gaussian, generally with larger $r_{0}$ and lower $Z_{0}$ than the RFC. Experimentally, the profile of the RFS is more difficult to measure accurately than that of the RFC, but on the other hand predictions are less sensitive to the exact profile than to the strength and size. The total GC excitation at any moment is the difference of center and surround excitations (Enroth-Cugell \& Lennie, 1975). A first-order simplification is that the RFS is radially symmetric and concentric with the RFC (see, however, Dawis et al., 1984; Troy, 1993).

\section{The waveform of the single-photon response in photoreceptors}

Photoreceptors respond linearly at low stimulus intensity or low contrast. When stimulated with brief, dim flashes of light, they give responses of constant waveform, with amplitude increasing in proportion to the number of photoisomerizations received [amphibian rods and cones: Baylor et al. (1979), Perry \& McNaughton (1991); reptile rods and cones: Baylor et al. (1974), cat rods: Tamura et al. (1989); primate rods and cones, including human rods: Baylor et al. (1984), Schnapf et al. (1990), Tamura et al. (1991), Kraft et al. (1993)]. As a first-order generalization, the waveforms of both rods and cones resemble the impulse response of a low-pass filter chain (Fuortes \& Hodgkin, 1964; Baylor et al., 1974). Departing from this type of description, there are two points of contention with major functional consequences: (1) To what extent does the falling phase even of "small" cone responses (especially in light-adapted states) overshoot the original baseline, producing a band-pass filtered frequency characteristic (Baylor et al., 1974; Daly \& Normann, 1985; Schnapf et al., 1990)? (2) In what manner do primate cones light-adapt (see e.g. Shapley et al., 1993)? In other respects, the frequency response derived by different mathematical formulations differs rather little. 
Thus, we take as our point of departure a filter-chain description of the photoreceptor dim-flash response (the Poisson formulation: Baylor et al., 1974). Firstly, it unquestionably describes rod responses well. Secondly, the activation stages of phototransduction are similar in rods and cones (e.g. Yau, 1994): thus possible deviations of cone responses from this model, connected with the falling phase, may justifiably be introduced as second-order effects. Thirdly, it gives simple analytical expressions for both the amplitude and phase of modulation responses. Fourthly, it is characterized by a single time parameter, which allows a generalized presentation of the model in terms of dimensionless normalized variables (see below).

The Poisson impulse response time course $f(t)$ (peak amplitude normalized to unity) is given by

$$
f(t)=C_{P} t^{n-1} e^{-\alpha t}, \quad C_{P}=[e \alpha /(n-1)]^{n-1}
$$

The normalizing factor $C_{P}$ has no special significance; it is the inverse of the peak response amplitude prior to normalization ( $t_{p}^{n-1} e^{-\alpha t_{p}}$, where $t_{p}$ is time to peak).

The parameter $n$ determines the waveform: with increasing $n$ the "latency" increases and both rise and decay become sharper (decreasing the ratio of half-width to time-to-peak). The response also becomes more symmetrical (Gaussian-like). A single parameter, in eqn. (3) expressed as a reciprocal time constant $\alpha$, captures the absolute time scale.

The integration (summation) time of the impulse response is

$$
t_{i}=\int_{0}^{\infty} f(t) \mathrm{d} t
$$

For Poisson kinetics, $t_{i}$ is

$$
t_{i}=C_{P}(n-1) ! / \alpha^{n}=[e /(n-1)]^{n-1}(n-1) ! / \alpha
$$

\section{The transmission delay of center and surround signals}

Signals initiated in photoreceptors take time to travel to the ganglion cell. In a constant state of adaptation, the time for transmission can be considered as independent of the size of the signal (Donner, 1989; Donner et al., 1995). Although retinal transmission is physiologically complex and will only partly produce pure delays in a technical sense (cf. Chen \& Freeman, 1989), it is a reasonable first-order approximation to treat the overall result, from the viewpoint of the ganglion cell, as a fixed delay (see further in Discussion). However, delays are different for the center and surround pathways. It has been directly documented in flash experiments on many species that the surround signal is delayed relative to the center signal (Nye \& Naka, 1971; Werblin \& Copenhagen, 1974; Enroth-Cugell \& Lennie, 1975; Winters \& Hamasaki, 1976; Miller \& Dacheux, 1976; Donner, 1981 ), and the surround pathway is generally thought to involve an extra sign-inverting interneuron. Here we assume that center and surround response kinetics differ in no other way. In psychophysics, the attenuation of low temporal frequencies has often been modelled in the easiest way, by assuming that the surround attenuates high temporal frequencies more strongly than the center (e.g. Watson, 1986). However, this notion goes against physiological evidence (Frishman et al., 1987 and below) and even predicts GC modulation sensitivities less well (Derrington \& Lennie, 1982; Enroth-Cugell et al., 1983).

\section{The model}

\section{Stimuli}

The input signal here is the light distribution at the level of photoreceptor outer segments. For aperiodic stimulation, typical spatial patterns are full fields and circular spots, bars and borders of different dimensions. Typical temporal waveforms are square-wave pulses of different durations (brief flashes, long steps). Among periodic stimuli, sine-wave modulation occupies a special place for several good reasons: (1) all other waveforms can be synthesized from sinusoids; (2) for a drifting sine-wave, modulation in the spatial and temporal dimensions are separable [see eqn. (10)]; (3) when stimulation is done through the intact eye, optical blurring will only decrease the modulation depth, but will leave the sinusoidal shape and spatial frequency of the retinal pattern unaffected. Any other waveform will be distorted by optical blurring.

The standard spatial pattern is a one-dimensional grating where intensity varies sinusoidally at spatial frequency $f_{s}$ $\left(\mathrm{m}^{-1}\right)$. The distance between stripes is the wavelength $\lambda=1 / f_{s}$. The purely temporal counterpart is sine-wave flicker at frequency $f(\mathrm{~Hz})$. Sine-wave gratings drifting at velocity $v=f / f_{s}$ produce sinusoidal modulation in both space and time.

\section{The spatial modulation factor}

The intensity modulation $I(x, t)$ of a vertical sine-wave grating drifting perpendicularly to the stripes is given by

$$
I(x, t)=I_{0}[1+m \cos (k x-\omega t)]
$$

$x$ is the coordinate along the retina with zero at the midpoint of the RFC. $I_{0}$ is the average intensity of the light falling on the retina [photons $\mathrm{m}^{-2} \mathrm{~s}^{-1}$ ] $m$ is the modulation depth $(1>$ $m>0$ ), $k$ is the (circular) wave number $2 \pi f_{s}$, and $\omega$ is the angular velocity $2 \pi f$. The pattern moves with velocity $v=$ $f / f_{s}=\omega / k$. When $k=0$, eqn. (6) represents pure full-field temporal flicker and when $\omega=0$ it describes a stationary grating. A stationary grating whose contrast is sinusoidally reversed, $I_{0}[1+m \cos (k x) \cos (\omega t)]$ (a contrast-reversing grating), can be thought of as the sum of two waves moving in opposite directions (wave numbers $k$ and $-k$, amplitudes $I_{0} / 2$ when the RF midpoint is at $x=0$ ). Thus, the stimulation produced by such a grating is as given by eqn. (6).

In the present analysis, $m$ always refers to the modulation at the level of photoreceptor outer segments. Note that if the stimulus modulation is $m_{0}$ and $L$ is the optical line spread function, the ratio $\mathrm{m} / \mathrm{m}_{0}$ is the optical modulation transfer function,

$$
m / m_{0}=\int_{-\infty}^{\infty} \cos (k x) L(x) \mathrm{d} x
$$

We define the excitation intensity $I_{e}$ in the RFC as

$$
I_{e}(t)=Q \int_{-\infty}^{\infty} \int_{-\infty}^{\infty} I(x, y, t) z(x, y) \mathrm{d} x \mathrm{~d} y
$$

where $Q$ is quantum efficiency in the midpoint of the RFC (the probability that an impinging photon produces a quantal excitation) and $I$ is the light intensity (photons $\mathrm{m}^{-2} \mathrm{~s}^{-1}$ ). Thus, the unit of $I_{e}$ is quantal responses per second reaching the GC 
through the center pathway. The excitation intensity caused by a constant light intensity $I_{0}$ is $I_{e 0}=Q I_{0} A_{i}$ [cf. eqn. (2)]. Thus, the excitation intensity for a sinusoidal light distribution [eqn. (6)] is

$$
I_{e}(t)=I_{e 0}+m I_{e 0} A_{i}^{-1} \int_{-\infty}^{\infty} \int_{-\infty}^{\infty} \cos (k x-\omega t) z(x, y) \mathrm{d} x \mathrm{~d} y
$$

Observing that $\cos (k x-\omega t)=\cos (k x) \cos (\omega t)+\sin (k x) \sin (\omega t)$ and $\iint \sin (k x) z \mathrm{~d} x \mathrm{~d} y=0$ [since $\sin (k x) z(x, y)=-\sin [k(-x)]$. $z(-x, y)$ for every $x$ ], eqn. (9) becomes

$$
\begin{aligned}
I_{e}(t)= & I_{e 0}+m I_{e 0} \\
& \times\left[A_{i}^{-1} \int_{-\infty}^{\infty} \int_{-\infty}^{\infty} \cos (k x) z(x, y) \mathrm{d} x \mathrm{~d} y\right] \cos (\omega t)
\end{aligned}
$$

Thus, $I_{e}-I_{e 0}$ can be expressed as a product of temporal and spatial factors, i.e. the temporal and spatial factors are separable.

The spatial factor in brackets, hereafter denoted $G$, is dimensionless, and depends only on what we shall term the normalized spatial frequency $F_{s}=r_{0} f_{s}$ (dimensionless). Then

$$
\begin{aligned}
I_{e}(t) & =I_{e 0}+m I_{e 0} G \cos (\omega t) \\
G & =G\left(F_{s}\right)=A_{i}^{-1} \int_{-\infty}^{\infty} \int_{-\infty}^{\infty} \cos (k x) z(x, y) \mathrm{d} x \mathrm{~d} y
\end{aligned}
$$

For a Gaussian RF, the function $G$ is (Appendix 1)

$$
G=e^{-\pi^{2} F_{s}^{2}}
$$

For $F_{s}=0$ (spatially homogeneous field), $G=1$. As $F_{s}$ increases, $G$ decreases monotonically to zero (see Fig. 2).

\section{The temporal modulation factor}

It is assumed that the GC response $U_{s}$ to single photons absorbed in different parts of the RFC have the same amplitude $U_{1}$ and the same time course $f(t)$ :

$$
U_{s}=U_{1} f(t), \quad 0<f(t)<1
$$

It is just a matter of convenience to assume constant $U_{1}$ at the ganglion cell and let the $z$ function take care of the variable gains of transmission in the different parts of the RFC. (This choice would be impermissible if we were considering quantal noise.)

The GC response at any time $t$ is the sum of the responses $U_{s}$ to photons absorbed at different times $t^{\prime}$ shortly before $t$. Assuming that many photons are absorbed per integration time, the response may be presented as a convolution integral

$$
U(t)=\int_{-\infty}^{t} U_{1} f\left(t-t^{\prime}\right) I_{e}\left(t^{\prime}\right) \mathrm{d} t^{\prime}
$$

An easier integral is obtained by substituting the variable $\tau=$ $t-t^{\prime}$ :

$$
U(t)=U_{1} \int_{0}^{\infty} f(\tau) I_{e}(t-\tau) \mathrm{d} \tau
$$

If the excitation intensity is constant $I_{e 0}$ [eqn. (11) with $m=0$ ] the corresponding constant response is

$$
U_{0}=U_{1} Q A_{i} t_{i} I_{0}=U_{1} I_{e 0} t_{i}
$$

Eqn. (16) gives the response to any excitation intensity. For a drifting sine-wave, $I_{e}$ is given by eqn. (11) and the response is

$$
U(t)=U_{0}+m U_{1} I_{e 0} G \int_{0}^{\infty} f(\tau) \cos (\omega \tau-\omega t) \mathrm{d} \tau
$$

Since $\cos (\omega \tau-\omega t)=\cos (\omega \tau) \cos (\omega t)+\sin (\omega \tau) \sin (\omega t)$, the integral can be represented in the form

$$
\begin{aligned}
h(t) & =h_{1} \cos (\omega t)+h_{2} \sin (\omega t)=h_{0} \cos \left(\omega t+\delta_{h}\right) \\
h_{1} & =\int_{0}^{\infty} f(\tau) \cos (\omega \tau) \mathrm{d} \tau \\
h_{2} & =\int_{0}^{\infty} f(\tau) \sin (\omega \tau) \mathrm{d} \tau
\end{aligned}
$$

Thus, $h(t)$ varies sinusoidally with frequency $f$ and amplitude

$$
h_{0}=\sqrt{h_{1}^{2}+h_{2}^{2}}
$$

The modulation response, with amplitude $\Delta U$, is [eqns. (18) and (19)]

$$
U(t)-U_{0}=\Delta U \cos \left(\omega t+\delta_{c}\right), \quad \Delta U=m U_{1} I_{e 0} G h_{0}
$$

The phase shift between the sinusoidal stimulus $I(t)-I_{0}$ and $h(t)$ is $\delta_{h}=-\tan ^{-1}\left(h_{2} / h_{1}\right)$. Including a constant delay for signal transmission through the retina, $d_{c}$ (see the section Physiological elements above), the total phase shift between the stimulus and the cell's response is

$$
\delta_{c}=\delta_{h}-\omega d_{c}=-\tan ^{-1}\left(h_{2} / h_{1}\right)-\omega d_{c}
$$

For Poisson kinetics [eqn. (3)], calculation of $h_{0}$ and $\delta_{h}$ is straightforward (Appendix 2). In this case, it is convenient to define a dimensionless factor $H=h_{0} / t_{i}$ that depends only on the dimensionless normalized frequency $F=t_{i} f$ :

$$
\begin{aligned}
& H=H(F)=\left[1+N(n)^{2} F^{2}\right]^{-n / 2} \\
& N=\frac{2 \pi}{\alpha t_{i}}=\frac{2 \pi(n-1)^{n-1}}{e^{n-1}(n-1) !}
\end{aligned}
$$

The amplitude of the sinusoidal response can then be written as $\Delta U=m U_{0} G H$ [eqns. (17) and (23)].

The normalized response amplitude is

$$
\Delta U /\left(m U_{0}\right)=H G
$$

The phase shift is now simply

$$
\delta_{c}=\delta_{c}(F)=-n \tan ^{-1}(N F)-2 \pi D_{c} F
$$

$D_{c}$ is the normalized dimensionless RFC delay $d_{c} / t_{i}$. For example, the typical rod value $n=4$ gives 


$$
H \approx \frac{1}{\left(1+2 F^{2}\right)^{2}}, \quad \delta_{h} \approx-4 \tan ^{-1}(1.41 F)
$$

\section{Center/surround antagonism}

Assume that the RFC (indexed $c$ ) has effective radius $r_{0 c}$ and the RFS (indexed $a$ for antagonistic) radius $r_{0 a}$ [eqn. (2)]. The ratio $r_{0 a} / r_{0 c}$, which by definition is larger than one, is denoted $R$. When the $G$ factor of the RFC is $G_{c}=G\left(F_{s}\right)$, that of the RFS is $G_{a}=G\left(R F_{s}\right)=\exp \left(-\pi^{2} R^{2} F_{s}^{2}\right)$. The RFS has larger area, so (assuming constant quantum efficiency $Q$ ), the excitation intensity due to a homogeneous field, $I_{e 0}$ [eqn. (10)], is larger for the surround. On the other hand, RFS peak sensitivity $Z_{0}$ is smaller, producing smaller photon response amplitudes $U_{1}$ at the ganglion cell. The "strength ratio" $K=\left(U_{1} I_{e 0}\right)_{R F S} /$ $\left(U_{1} I_{e 0}\right)_{R F C}$ reflects the balance of these two factors. The GC is balanced if $K=1$.

Surround responses are assumed to have the same waveform as center responses. When the stationary response of the center is $U_{0}$ [eqn. (17)], that of the surround is then $K U_{0}$. The transmission delay of the surround is longer by the amount $d$. The GC response at any moment is the sum of the contributions from the RFC and the RFS. The resultant modulation response is

$$
\begin{aligned}
U(t)= & U_{0}+m U_{1} I_{e 0} G_{c} h_{0} \cos \left(\omega t+\delta_{c}\right)-K U_{0} \\
& -m U_{1} K I_{e 0} G_{a} h_{0} \cos \left(\omega t+\delta_{c}+\omega d\right)
\end{aligned}
$$

This consists of a stationary part $\Delta U_{0}=(K-1) U_{0}$ on which a sum of two sinusoids with phase difference $\omega d$ is superimposed.

The two sinusoids can be treated as phase vectors (phasors) $\Delta U_{c}$ and $\Delta U_{a}$ having a phase difference $\pi-\omega d$, as illustrated in Fig. 1. (This extended version of the DOG model was originally proposed by Enroth-Cugell et al. [1983], who termed it the Gaussian center-surround model.) The vector sum is a sinewave with the same frequency,

$$
U(t)-\Delta U_{0}=\Delta U \cos (\omega t+\delta)
$$

The amplitude $\Delta U$ and the phase shift $\delta$ of the resultant wave are

$$
\begin{aligned}
\Delta U & =m U_{1} I_{e 0} h_{0} \sqrt{G_{c}^{2}+K^{2} G_{a}^{2}-2 K G_{c} G_{a} \cos (\omega d)} \\
\delta & =\delta_{c} \pm \cos ^{-1} \frac{G_{c}-K G_{a} \cos (\omega d)}{\sqrt{G_{c}^{2}+K^{2} G_{a}^{2}-2 K G_{c} G_{a} \cos (\omega d)}}
\end{aligned}
$$

The plus sign holds for $0<\omega d<\pi$ and the minus sign for $\pi<\omega d<2 \pi$.

For Poisson kinetics it is convenient to define a normalized responsivity $\Delta U /\left(m U_{0}\right)$, where $U_{0}$ is the average response of the RFC given in eqn. (17). We obtain

$$
\Delta U /\left(m U_{0}\right)=H \sqrt{G_{c}^{2}+K^{2} G_{a}^{2}-2 K G_{c} G_{a} \cos (2 \pi D F)}
$$

Thus, the normalized responsivity to sinusoidal modulation, $\Delta U /\left(m U_{0}\right)$, depends only on five dimensionless quantities: the normalized frequencies $F$ and $F_{s}$ of the stimulus light and three RF parameters: the strength ratio $K$, the radius ratio $R$, and the normalized surround-center delay $D=d / t_{i}$.

A


B

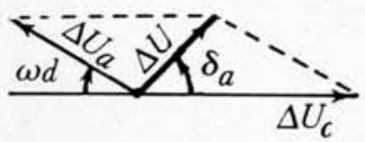

C

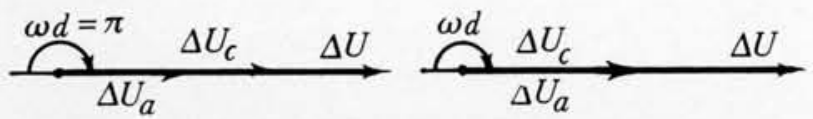

Fig. 1. The response $\triangle U$ of a GC with DOG RF represented as a vector sum of RFC and RFS phase vectors $\Delta U_{c}$ and $\Delta U_{a}$. (A-C): RFS/ RFC strength ratio $K=0.6$. (D-F): $K=1$ (balanced RF). (A,D): $\omega=0$; (B,E): $\omega d=0.5 \mathrm{rad} ;(\mathrm{C}, \mathrm{F}): \omega d=\pi . \delta_{a}$ is the contribution of the RFS to the phase shift.

\section{Responses to spatial modulation}

The success of the DOG model in predicting GC responses to spatial sine-wave gratings is a well-established fact of visual science (see, e.g. Enroth-Cugell \& Robson, 1984; Troy, 1993). We dwell on the spatial modulation response here only for comparison with the temporal modulation response, and as a preliminary to the discussion of spatio-temporal interactions.

To isolate spatial responsivity, we consider the normalized response amplitude at a very low temporal frequency. Then $h_{0}$ is constant [eqns. $(20-22)$ ] and $\cos (\omega d) \approx 1$. Therefore, center and surround signals are in opposite phases and $\Delta U$ is proportional to $G_{c}-K G_{a}$ (in a balanced GC, $G_{c}-G_{a}$ ). The three solid curves in Fig. 2 trace, for a balanced GC with $R=r_{0 a} / r_{0 c}=3$, the normalized response of the center $\left(G_{c}\right)$, the surround $\left(G_{a}\right)$, and the resultant of these $\left(G_{c}-G_{a}\right)$. The same curves are shown on linear scales in Fig. 2A and in the more customary logarithmic presentation in Fig. 2B.

Center-surround organized cells of course always respond poorly to homogeneous illumination, and in a balanced DOG GC responsivity is exactly zero for zero spatial frequency (at $F_{s}=0$ in Fig. 2A). For high $F_{s}$, on the other hand, the normalized response is approximately $G_{c}$ (unaffected by the surround) provided that $R \gg 1$. Between these extremes, response attenuation due to surround antagonism is a monotonically decreasing function of $F_{s}$.

In Fig. 2, the effect of the surround (a subtractive mechanism) has also been represented in terms of a multiplicative "spatial attenuation factor" $P_{s}=\left(G_{c}-G_{a}\right) / G_{c}$ (dot-dashed line). The purpose is to parallel the formalism used in recent psychophysical work on grating detection (Rovamo et al., 1993, 1994), where a high-pass filter identified as a modulation transfer function of the visual pathways $P_{\mathrm{MTF}}$ has been introduced to take care of the attenuation of low spatial frequencies. The authors find that the attenuation, which they tentatively ascribe to lateral antagonism, decreases linearly with increasing $f_{s}$ (i.e. $P_{\mathrm{MTF}} \propto$ $F_{s}$ ). As seen from Fig. 2, $P_{s} \propto F_{s}$ is indeed a good approximation, but only over a limited range. $P_{s}$ saturates at the spatial 


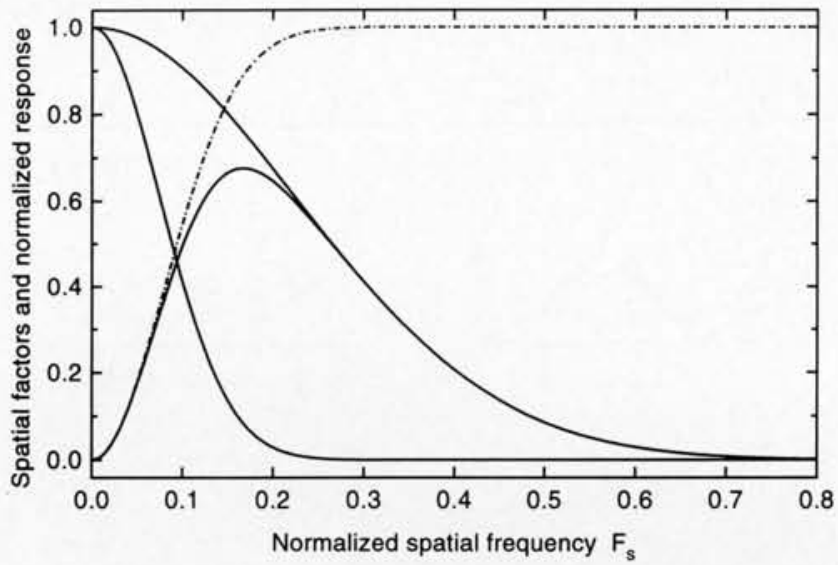

A

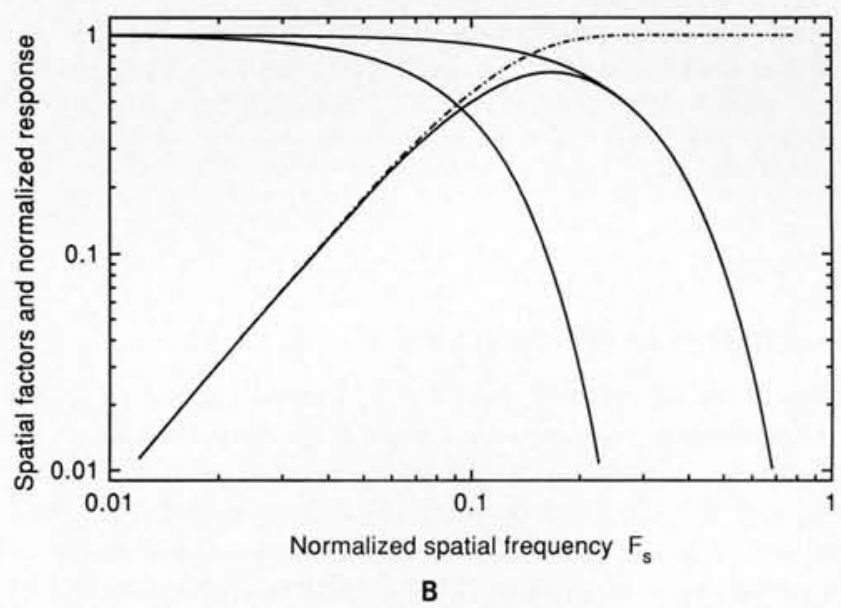

Fig. 2. The response of a balanced DOG RF with $R=3$ to "stationary" gratings (see text) at different normalized spatial frequencies $F_{s}=r_{0 c} f_{s}$. The three solid curves give (1) the normalized center response $G_{c}=\exp \left(-\pi^{2} F_{s}^{2}\right)$ (upper monotonic curve), (2) the normalized surround response $G_{a}=\exp \left(-R^{2} \pi^{2} F_{s}^{2}\right)$ (lower monotonic curve), and (3) the normalized resultant response $G_{c}-G_{a}$. The dot-dashed line gives the spatial attenuation factor $P_{s}=\left(G_{c}-G_{a}\right) / G_{c}$. (A), linear scales; and (B), logarithmic scales.

frequencies to which the GC is most sensitive. In this, it differs not only from the $P_{\mathrm{MTF}}$ of Rovamo et al. $(1993,1994)$ but also from the low-frequency attenuation factor associated with temporal modulation transfer (see below).

\section{Responses to temporal and spatio-temporal modulation assuming that photoreceptors follow Poisson kinetics}

The temporal response

To isolate temporal responsivity, it is assumed that there is no spatial modulation $\left(F_{s}=0\right)$, hence $G_{c}=G_{a}=1$. The normalized response to sinusoidal flicker is then [eqn. (33)]

$$
\Delta U /\left(m U_{0}\right)=H \sqrt{1+K^{2}-2 K \cos (2 \pi D F)}
$$

Fig. 3 summarizes the main determinants of temporal responsivity in a balanced GC on linear scales (Fig. 3A) and on logarithmic scales (Fig. 3B). The solid curve traces the factor $H$,

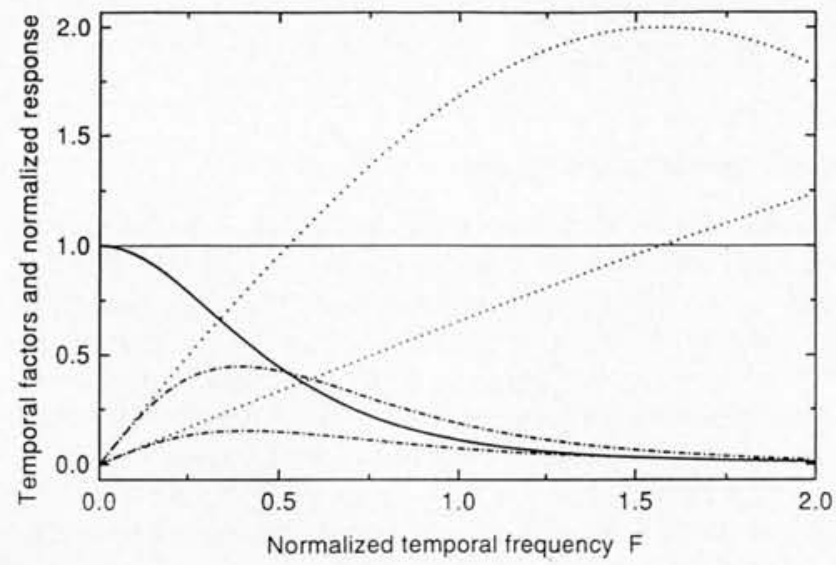

A

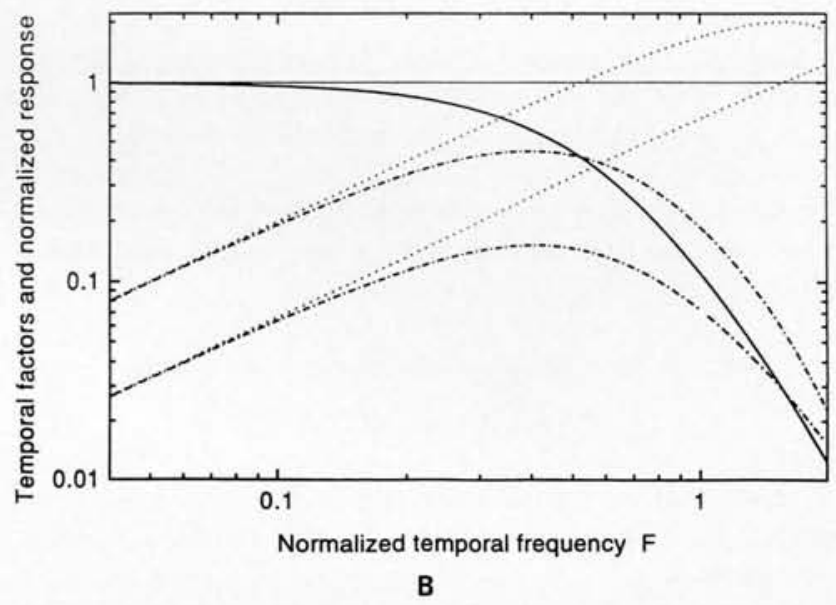

Fig. 3. The response of a balanced DOG RF to spatially homogeneous flicker at different normalized temporal frequencies $F=t_{i} f$. Poisson kinetics with $n=4$. Both RFC and RFS have the same $t_{i}$ but the surround response arrives at the GC with a delay $D=d / t_{i}$. The solid line shows the normalized response of center or surround alone, $H=(1+$ $\left.2 F^{2}\right)^{-2}$. The two dotted lines show the temporal attenuation factor $P_{t}=\sqrt{2-2 \cos (2 \pi D F)}$ for $D=1 / \pi \approx 0.318$ (upper line) and $D=$ $1 /(3 \pi) \approx 0.106$ (lower line), respectively. The dot-dashed lines show, for these two $D$ values, the normalized resultant response $\Delta U /\left(m U_{0}\right)=$ $H \sqrt{2-2 \cos (2 \pi D F)}$. (A), linear scales; and (B), logarithmic scales.

the normalized response of center or surround alone. The two dotted curves show the square root factor in eqn. (34) for two values of the surround-center delay $D$. The square-root factor is a correlate of a psychophysical "temporal modulation transfer function of the visual pathways" (temporal $P_{\mathrm{MTF}}$ ) (Rovamo et al., 1995) and we shall denote it $P_{t}$. Obviously, $P_{t}$ depends strongly on $D$ : the two examples in Fig. 3 correspond to $D=$ $1 / \pi$ (upper) and $D=1 /(3 \pi)$ (lower).

At very low frequencies the phases of $\Delta U_{c}$ and $\Delta U_{a}$ are opposite, whereby $P_{t}=1-K$, i.e. a balanced GC $(K=1)$ does not respond at all. With increasing $F$, the phase difference deviates increasingly from $\pi$, and $P_{t}$ increases near linearly. At high frequencies, the surround phase lag grows so large that center and surround begin to interfere constructively $\left(P_{t}>1\right)$. At $F=1 /(2 D)$, the signals are in phase and $P_{t}=2$ in a balanced GC. For $D=1 / \pi, P_{t}$ peaks at $F=\pi / 2 \approx 1.57$, but for $D=$ $1 /(3 \pi)$ at $F=3 \pi / 2 \approx 4.71$, outside the figure. 
The dot-dashed curves in Fig. 3 show the resultant normalized responses $H P_{t}$, the higher one for $D=1 / \pi$ and the lower one for $D=1 /(3 \pi)$. The maxima lie at much lower frequencies than the $P_{t}$ maxima, because even at $F=1 / 2$ the function $H$ falls steeply and dominates the resultant. The smaller $D$ is, the larger will be the difference between the response peak and the $P_{t}$ peak. Physiologically, $D$ appears always to be $<1$ (see below).

To summarize, temporally as well as spatially, the subtractive mechanism of surround antagonism produces high-pass filtering such that signal attenuation in a certain range is inversely proportional to modulation frequency. In the temporal but not in the spatial domain, this range may extend far beyond the frequency of peak responsivity.

In a general case, spatial and temporal stimulus characteristics interact to determine the response [eqn. (33)]. It is then to be observed that effective attenuation by surround antagonism occurs only when both $F_{s}$ and $F$ are low.

\section{Comparison with physiology}

How well does the model predict recorded modulation responses when provided with parameter values derived from "aperiodic" experiments? We shall not reproduce the solid evidence that good fits to spatial modulation responses are provided by values that are consistent with RF profiles mapped by other techniques (see Introduction). Our main concern here is the temporal dimension of the spatio-temporal responsivity surface.

\section{The time parameters}

Regrettably, for neither rods nor cones of any mammalian species do we have all the numbers needed for (1) the absolute time scale of the photoreceptor response, (2) the transmission delay of the center pathway, and (3) the surround-center delay. For frogs, these are available, but instead modulation responsivity data is lacking. (Obtaining such data in frogs presents particular problems due to progressive desensitization of GCs under periodic stimulation: see Donner et al., 1991.) Table 1 collects relevant data from the literature. Although incomplete, it is extensive enough to allow some important generalizations.
(1) The absolute time scale of the quantal response of photoreceptors is represented by the time-to-peak $t_{p}$ of dim-flash responses. This is an easily measured parameter, and for a fixed response shape, it fully describes the time scale. Under Poisson kinetics, $t_{p}=(n-1) / \alpha$, and the parameter $t_{i}$ mainly used in the equations is thus related to $t_{p}$ via eqn. (5) which gives $t_{i} \approx 1.14 t_{p}$ for $n=6$ and $\approx 1.04 t_{p}$ for $n=7$. The mammalian values in Table 1 are quite consistent, indicating $t_{p}=30-50 \mathrm{~ms}$ for dark-adapted cones and $t_{p} \approx 200 \mathrm{~ms}$ for dark-adapted rods.

Photoreceptor responses accelerate with light adaptation. Elsewhere, we have presented evidence that time scales may be recalculated to any mean illumination $I_{0}$ by the simple rule $t_{p} \propto\left(I_{0} / I_{D}\right)^{-b}$, where $I_{D}$ is the "dark light" and $0.1<b<0.2$ depending on species and photoreceptor type (Donner et al., 1995). This rule holds over $I_{0}$ ranges extending approximately $3 \log$ units above $I_{D}$; beyond that, response acceleration saturates and is even reversed. For mammalian cones, we may assume $b=0.15$, which is the midpoint of the range and fits, e.g. the acceleration of human foveal vision with rising mean illumination.

(2) The transmission delay of the center pathway was determined from GC intensity-latency functions as described by Donner (1989) and Donner et al. (1995). The literature data used for the analysis (references given in Table 1) is of variable quality, hence the reliability of the estimates varies (in the order frog $>$ turtle $>$ monkey $>$ cat). These are best estimates, and we prefer not to give numerical confidence limits, which would convey a misleading impression of statistical treatment (where uncertainty in reality mainly depends on assumptions). We note that monkey and cat estimates intersect at $15 \mathrm{~ms}$. In frog, we have found that the delay shortens moderately (by ca. 30\%) with light adaptation (Donner et al., 1995). If this applies to mammals, too, cone delays could drop to $10 \mathrm{~ms}$ in light-adapted states. The large difference between rod and cone delays in monkey $(25 \mathrm{~ms})$, on the other hand, is consistent with the fact that rod signals are mediated by a different pathway (see e.g. Kolb, 1994). In this, mammals differ from amphibians.

(3) The surround-center delay is the most uncertain parameter, with no good mammalian data available from aperiodic experiments. For the present purpose, published studies are flawed by either too low resolution and/or failure to distin-

Table 1. Time parameters from flash/step experiments (dark-adapted) ${ }^{a, b}$

\begin{tabular}{|c|c|c|c|c|c|c|c|}
\hline & \multicolumn{4}{|c|}{$t_{p}$ of the photoreceptor dim-flash response (ms) } & \multicolumn{3}{|c|}{ Transmission delays (ms) } \\
\hline & \multicolumn{2}{|c|}{ Directly recorded } & \multicolumn{2}{|c|}{ From GC latencies } & \multicolumn{2}{|c|}{ Center pathway (RFC) } & \multirow[b]{2}{*}{ RFS - RFC } \\
\hline & Rod & Cone & Rod & Cone & Rod & Cone & \\
\hline Cat & $200^{(1)}$ & $?$ & $?$ & $30^{(2)}$ & ? & $15-30^{(2)}$ & $(0-40)^{(3.4)}$ \\
\hline Monkey & $190-230^{(5,6)}$ & $40-50^{(7)}$ & $230^{(8)}$ & $25^{(8)}$ & $40^{(8)}$ & $15^{(8)}$ & ? \\
\hline Human & $190^{(9)}$ & $35^{(10)}$ & $?$ & ? & $?$ & $?$ & ? \\
\hline Turtle $\left(19-22^{\circ} \mathrm{C}\right)$ & $300-500^{(11)}$ & $100-160^{(11)}$ & ? & $120-150^{(12)}$ & $?$ & $35-50^{(12)}$ & $?$ \\
\hline Frog $\left(10-12^{\circ} \mathrm{C}\right)$ & $1700-4000^{(13)}$ & $200-400^{(14)}$ & $1500-2500^{(15)}$ & $300-500^{(14)}$ & $120-200^{(13.15)}$ & $100-120^{(13)}$ & $30-40^{(16)}$ \\
\hline
\end{tabular}

\footnotetext{
${ }^{a}$ The determination of photoreceptor kinetics and transmission delays from GC data was based on analysis of intensity-latency functions as described in Donner (1989) and Donner et al. (1995). In the underlying filter-cascade models for photoreceptor responses, $n$ was always taken as 4 or 5 for rods and as 6 or 7 cones. All GC data refer to on-pathways.

bources: ${ }^{1}$ Tamura et al., 1989; ${ }^{2}$ Bolz et al., 1982; ${ }^{3}$ Winters \& Hamasaki, 1976; ${ }^{4}$ Enroth-Cugell \& Lennie, 1975; ${ }^{5}$ Baylor et al., 1984 ; ${ }^{6}$ Tamura et al., 1991; ${ }^{7}$ Schnapf et al., 1990; ${ }^{8}$ Gouras \& Link, $1966 ;{ }^{9}$ Kraft et al., 1993; ${ }^{10}$ Hood \& Birch, 1993 b; ${ }^{11}$ Baylor et al., 1974 ; ${ }^{12}$ Baylor \& Fettiplace, $1977 b ;{ }^{13}$ Donner et al., 1995; ${ }^{14} \mathrm{~K}$. Donner, S. Hemilä \& A. Koskelainen, unpublished; ${ }^{15}$ Donner, $1989 ;{ }^{16}$ Donner, 1981.
} 
guish between true delays and such time shifts that are caused by unequal excitation of the RFC and RFS (Enroth-Cugell \& Lennie, 1975; Winters \& Hamasaki, 1976). For the latter reason, the larger estimates ( $15-40 \mathrm{~ms})$ are almost certainly wrong, but on the experimental evidence the true value could lie anywhere between 0 and $10 \mathrm{~ms}$. A safe generalization (cf. the frog data in Table 1) would be that the surround-center delay is smaller than the full center-pathway delay. The latter in turn is smaller than $t_{i}$, hence the normalized surround-center delay $D=d / t_{i}<1$.

Temporal responsivity and phase in a photopic state: Inadequacy of pure Poisson kinetics

To test the model, we use the data of Frishman et al. (1987), who have published the most extensive study of temporal modulation responses in linear cat GCs (X-cells). Unlike Victor (1987), they make clear distinctions between (fairly) pure center responses and responses with various degrees of surround involvement. They also present data for several adaptation levels (see below).

We first consider a fixed photopic state of adaptation. The data displayed in Figs. 4C and 4D is taken from Fig. 3 of Frishman et al. (1987), summarizing modulation responses of 17 oncenter X-cells mainly at $340 \mathrm{~cd} \mathrm{~m}^{-2}$ mean illuminance. Fig. $4 \mathrm{C}$ shows responsivities: solid circles for center-isolating stimulation and open circles for "diffuse" stimulation that fully activates both center and surround. Each data point is the logarithmic mean of the responsivities of all 17 cells normalized to maximum 1. Panel (D) displays the phase data, as mean values for the center responses (solid circles) but as range bars for diffuse stimulation. (The latter data split into two branches at low frequencies, making it less appropriate to use means.)

To explain the center curves drawn in panels (C) and (D), we must first turn to panels (A) and (B) which illustrate three alternative forms of cone kinetics considered for a fit. These are shown in panel (A) as responses to sinusoidal modulation and in panel (B) as impulse responses. This diversion is neces-

Fig. 4. Model fit to cat GC temporal responsivities. (A) and (B) show the three different temporal response waveforms considered for a fit, (A) as modulation responses and (B) as impulse (flash) responses. The curves correspond to (1) Poisson kinetics with $n=5$ and $\alpha=220 \mathrm{~s}^{-1}$ (dot-dashed line); (2) "partly differentiated" Poisson kinetics, a $30 \%+$ $70 \%$ combination of the "direct" response and a response that has been high-pass filtered with time constant $120 \mathrm{~ms}$ (continuous line); and (3) the photocurrent response waveform recorded by Schnapf et al. (1990) in single monkey cones (dotted). The necessity to consider different waveforms is due to the fact that simple Poisson kinetics does not produce the low-frequency attenuation of pure center responses evident in the experimental data (C). (C), responsivity data and (D), phase data from Frishman et al. (1987: Fig. 3) fitted with the DOG model. Data points are averages of the experimental values for $17 \mathrm{X}$-cells: solid circles - RFC, open circles - RFC + RFS. The RFC + RFS phase data are represented by range bars, as the distribution at low frequencies split into two branches. Center parameters: "partly differentiated" Poisson kinetics with $n=7$ and $\alpha=450 \mathrm{~s}^{-1}, 30 \%$ "direct", $70 \%$ high-pass filtered with time constant $120 \mathrm{~ms}$. Normalized to unity maximum responsivity. The center transmission delay in the phase calculation was $d_{c}=$ 10 ms. DOG RF parameters: $G_{c}=G_{s}=1$ (no spatial modulation), $K=0.85, d=7 \mathrm{~ms}$, stimulation of the RFC assumed to be the same as for the "center" curve.



A
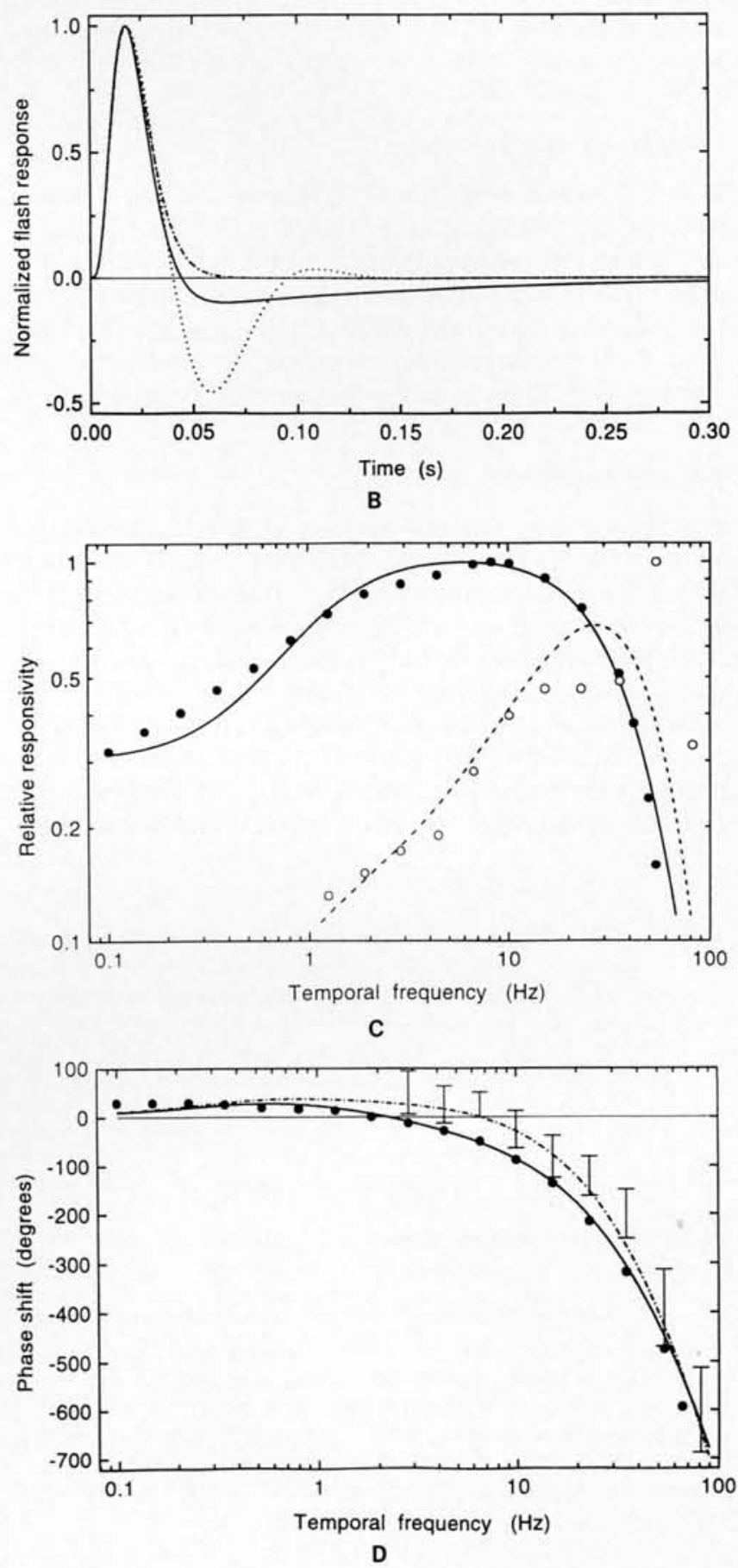
sitated by the immediately evident fact that pure Poisson kinetics as such (dot-dashed responsivity curve in panel A) cannot produce the low-frequency roll-off observed in center responsivity (solid circles in panel C). The remedy that first occurred to us was to replace the Poisson response with the actual biphasic (or multiphasic) impulse response recorded in isolated monkey cones by Schnapf et al. (1990) (dotted curves in panels A and B). This was not a good choice: the resulting responsivity is indeed a non-monotonic function of frequency, but otherwise bears little resemblance to the experimental curve. It thus appeared necessary to introduce some other mechanism. In experiments with injection of d.c.-current into turtle cones, Baylor and Fettiplace $(1977 a, b, 1979)$ have shown that the signal from cones to GCs is differentiated during retinal transmission. Also, Victor (1987) has argued that transmission in cat involves high-pass filtering not due to the RFS. We therefore postulated that the response reaching the GC is a composite of a Poisson response and a retinally $\mathrm{RC}$-filtered version of the same. The "partly differentiated Poisson" waveform shown in the figure could in principle equally well represent the shape of the cone impulse response, or it could arise as any combination of biphasic cone responses and retinal differentiation. The different physiological assignments make no mathematical difference.

To see the specific features associated with the shape of the negative overshoot as clearly as possible, we chose other parameters in panels A and B so that the positive part of all three responses should closely superpose. For both the responses based on Poisson kinetics, $n=5$ was therefore chosen. In the second one (full-drawn line), $70 \%$ of this was high-pass filtered with time constant $120 \mathrm{~ms}$. The modulation response corresponding to this partly high-pass filtered Poisson kinetics is seen to be qualitatively quite similar to the recorded "center" response in panel $\mathrm{C}$.

For the actual "center" fits (full-drawn curves in panels C and D), responsivities and phase shifts were calculated numerically [eqns. (19-24)] with Poisson parameters $n=7$ and $\alpha=$ $450 \mathrm{~s}^{-1}$ (corresponding to $t_{p}=13 \mathrm{~ms}$ ). (An analytic solution for responsivity is possible, but rather cumbersome.) The curves for diffuse stimulation (dashed curves in panels $\mathrm{C}$ and $\mathrm{D}$ ) show the predictions of the DOG model [eqns. (31) and (32)] for the same kinetics, assuming RF strength ratio $K=0.85$ and surroundcenter delay $d=7 \mathrm{~ms}$. Fitting the four curves in panels C and D involved no further adjustments. The vertical position of the "diffuse" responsivity curve is fixed by the requirement that RFC stimulation be equal for both curves. Clearly, most features of the experimental data are reproduced reasonably well. The main unexplained feature is the sharp responsivity peak appearing at $50-60 \mathrm{~Hz}$ under diffuse stimulation [but also to a lesser degree with more center-favoring stimuli, see Frishman et al. (1987)]. This is evidently due to some entirely different mechanism, e.g. some form of neural resonance as indeed suggested by the authors. It is noteworthy that the peak is small or absent at somewhat lower adaptation levels and has not been observed in psychophysical measurements of temporal resolution in cat or humans. A minor discrepancy is that even center sensitivity at the highest frequencies falls a little more steeply than the model curve, indicating some additional low-pass filtering proximal to photoreceptors.

We should like to emphasize that all of the parameter values of the fit are consistent with those in Table 1, observing the high mean luminance (corresponding to some $4 \times 10^{3} \mathrm{td}$, more than $3 \log$ units above cone dark light, implying that cone $t_{p}$ is expected to be $10-15 \mathrm{~ms}$ ). Thus, our general conclusion is that a good fit to the main characteristics of temporal modulation responses (responsivity and phase functions for both "center" and "diffuse" stimulation, save for the $50-60 \mathrm{~Hz}$ peak) is achieved with the same parameter values that fit results from aperiodic experiments.

\section{Dependence on the state of adaptation}

Frishman et al. (1987) studied modulation responses at three different levels of mean illumination: "photopic" (most often $340 \mathrm{~cd} \mathrm{~m}^{-2}$, in a few cases 200 or $440 \mathrm{~cd} \mathrm{~m}^{-2}$ ), "midmesopic" $\left(2.8 \mathrm{~cd} \mathrm{~m}^{-2}\right)$, and "high scotopic" (light-adapted rods, 0.021 $\mathrm{cd} \mathrm{m}^{-2}$ ). In their Table 1, they summarize mean values for some crucial parameters of the responsivity and phase functions at each illumination level. Most of the changes can be given clear physiological interpretations:

(1) The dominant effect, shifting of the responsivity curve to higher frequencies at higher mean illumination, follows directly from photoreceptor response acceleration. As "photopic" was typically $2.1 \mathrm{log}$ units higher than "midmesopic" (see above) and $t_{p} \propto I_{0}^{-0.15}$ (Donner et al., 1995), cone responses would be faster by a factor of 2 or more in the photopic situation. Indeed, the "center" sensitivity maximum was found to shift from $2.9 \pm 0.6$ to $6.3 \pm 2.6 \mathrm{~Hz}$ on average ( \pm s.D., data from four midmesopic and 17 photopic cells).

(2) The mean slope of the high-frequency roll-off was $-5.5 \pm$ 1.1 in the midmesopic and $-6.7 \pm 1.3$ in the photopic state, both basically consistent with cone kinetics (Poisson with $n=$ 6 or 7), but $-2.6 \pm 2.2$ in the "high scotopic" state, basically consistent with rod kinetics (Poisson with $n=4$ ). Again, the general agreement with photoreceptor modulation responses does not of course exclude some additional low-pass filtering more proximally in the retina. (This could, for example, explain why the photopic slope, extending to higher frequencies, is steeper than the midmesopic slope).

(3) The low-frequency roll-off of center responsivity also became sharper with higher mean illumination. The slope values given by Frishman et al. (1987) are photopic 0.42 , midmesopic 0.39 , and high scotopic 0.28 . Thus, high-pass filtering (signal differentiation) becomes more pronounced at higher illumination levels. Changes of this kind could be achieved in several ways. Not to bring in more degrees of freedom than necessary, we choose to account for them only by increasing the proportion of high-pass filtered to unfiltered cone response reaching the GC (see above). As illustrated in Fig. 5, this is sufficient to provide the range of change required.

Fig. 5 summarizes adaptational changes in center responses according to the model, highlighting distinguishing factors rather than optimizing parameters to precisely fit experimental data (which would as such be easy). Relative responsivities were calculated using eqn. (22). "Dark-adapted scotopic" responsivity is based on the Poisson response with $n=4, \alpha=15 \mathrm{~s}^{-1}$ (i.e. $t_{p}=$ $200 \mathrm{~ms}$, cf. rods in Table 1). "Photopic" responsivities were calculated for basically the same kinetics $\left(n=7, \alpha=450 \mathrm{~s}^{-1}\right)$ as the fit of Fig. 4, but increasing the proportion of the high-pass filtered part to $100 \%$ to clearly show the effect of this factor. "Midmesopic" responsivities were obtained by slowing down photoreceptor response kinetics by half (see above) and modulating the degree of retinal differentiation $\left(n=7, \alpha=225 \mathrm{~s}^{-1}\right.$ and the high-pass filtered part of the response $60 \%$ ). Being displayed as relative responsivities, all curves in Fig. $5 \mathrm{~A}$ have maximum 1. In absolute terms, scotopic responsivities would be lower (Frishman et al., 1987). 


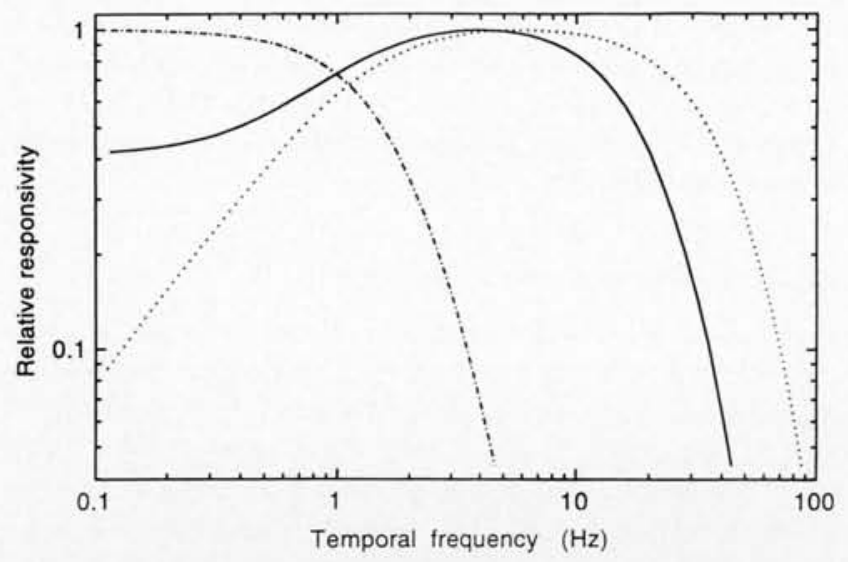

A

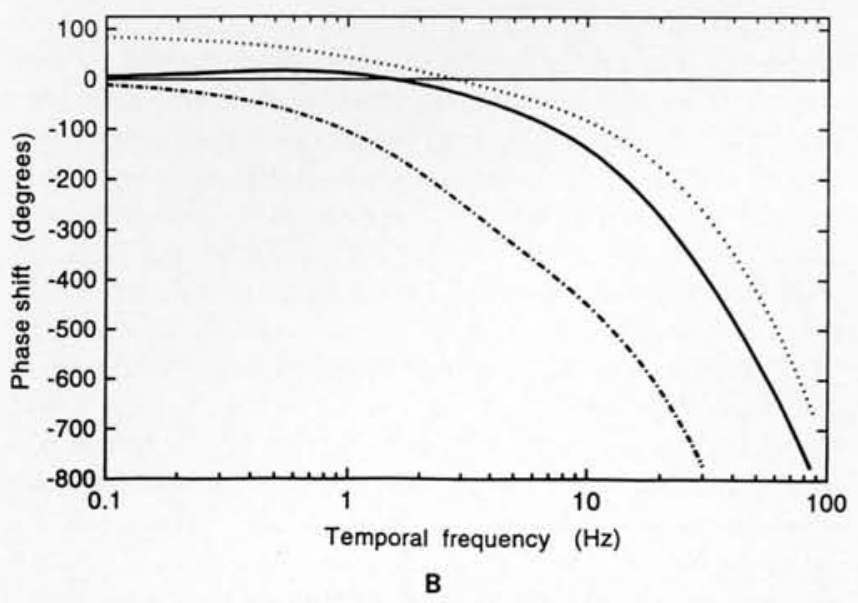

Fig. 5. Model simulation of the effects of light adaptation on RFC responsivity and phase. (A), responsivities; and (B), phase shifts. The dot-dashed line represents the dark-adapted state (rod kinetics): Poisson response with $n=4, \alpha=15 \mathrm{~s}^{-1}$, and $d_{c}=40 \mathrm{~ms}$. The solid line represents the midmesopic state: $n=7, \alpha=225 \mathrm{~s}^{-1}, 60 \%$ of the Poisson response high-pass filtered with the time constant $120 \mathrm{~ms}$, and $d_{c}=10 \mathrm{~ms}$. The dotted line represents the photopic state: $n=7, \alpha=$ $450 \mathrm{~s}^{-1}$, the Poisson response high-pass filtered with the time constant $120 \mathrm{~ms}$, and $d_{c}=10 \mathrm{~ms}$.

\section{Phase}

Frishman et al. (1987) found that the phase lag of the center response depended linearly on temporal frequency from about $2 \mathrm{~Hz}$ upwards. The slope of the straight line (in a linear plot, measured as cycles per $\mathrm{Hz}$, equivalent to $\mathrm{Hz}^{-1}$ or s) decreased with increasing mean luminance. The mean values given by Frishman et al. (1987) are $24 \mathrm{~ms}$ in the photopic state (17 cells), $33 \mathrm{~ms}$ in the midmesopic state (11 cells), and $77 \mathrm{~ms}$ in the high scotopic state (three cells). These values refer to "peak" stimuli (stimulating not only the RFC but to a substantial degree also the RFS) and were measured over the interval $2-80 \mathrm{~Hz}$ in the photopic and midmesopic states and over the interval $2-30 \mathrm{~Hz}$ in the high scotopic state.

For pure center responses, perfectly straight lines are expected if the phase shift is wholly due to a pure delay $\left(d_{c}\right)$, whereby the slopes are equal to $d_{c}$. The model phase curves in Fig. 5 would not be quite straight when transferred to linear coordinates, but would be a little steeper in the low-frequency end. This is because the phase lag at low frequencies is dominated by photoreceptor low-pass filtering but at high frequencies by the transport delay (see Discussion). Increasing the phase contribution of the surround will make the resultant phase functions increasingly linear.

The mean slopes of the model phase curves in Fig. 5 over the relevant frequency ranges are photopic 25 , midmesopic 28 , and scotopic $58 \mathrm{~ms}$. Thus, the basic tendency that the slope decreases with increasing mean luminance agrees with Frishman et al. (1987), and the photopic value happens to be almost identical. Although an exact agreement between these "center" phase curves and the "peak" curves of Frishman et al. (1987) should not be expected, we would like to point out that only minor parameter adjustments would be needed to achieve full coincidence in the midmesopic and scotopic states as well: it would be enough to increase $d_{c}$ to $18 \mathrm{~ms}$ and $60 \mathrm{~ms}$, respectively (but see Discussion regarding the contributions of other factors to response phase). Observing that all of the midmesopic and scotopic recordings were done in the optic tract so that the actual retinal delays are smaller by about $4 \mathrm{~ms}(14$ and $56 \mathrm{~ms})$, the values are broadly consistent with the estimates from aperiodic experiments (Table 1).

\section{Discussion}

\section{Scope of the model}

Our concern has been to identify and factor out major physiological effects, paying particular attention to the absolute parameter values needed to fit aperiodic and periodic results. In this, our approach is very different from a type of ad hoc modelling (often involving similar mathematical structures) designed to reproduce every minor feature of experimental data. We show that current knowledge of photoreceptor responses and photoreceptor-to-GC transmission derived from flash/step experiments when integrated into the DOG model explains the main characteristics of GC modulation responses not only in a particular state of adaptation, but also in their dependence on mean luminance.

Two effects emerging at high temporal frequencies $(50-60 \mathrm{~Hz})$ remain unexplained: (1) a sharp sensitivity peak observed under modulation activating both RFC and RFS (Fig. 4C) and (2) a moderate expansion of the RFC (Frishman et al., 1987). Other unexplained features are some excessive steepness in the highfrequency roll-off and some discrepancies in the shape of the low-frequency roll-off (Fig. 4C). It would as such be a trivial exercise to introduce and fine-tune additional (moreover, quite plausible) model elements to bring predictions in close coincidence with the data on these points as well (resonance circuits, high-pass characteristics of inter-photoreceptor coupling as described by Detwiler et al. [1980], low-pass filtering proximal to photoreceptors, multiple-stage high-pass filtering). This, however, goes beyond the objectives of the present paper.

A particular act of parsimony here was to neglect possible complications of cone response waveform and take Poisson kinetics as the point of departure. There could in principle be differentiation both in cones and at several stages in the retinathe model cannot tell where or how the "partly differentiated" Poisson response is generated. What we do know is that appropriate waveforms have not been recorded in cones. Moreover, recent photovoltage recordings from dark-adapted monkey cones in situ support our assumption that dim-flash responses 
are not biphasic (Schneeweis \& Schnapf, 1995). This is also consistent with the essentially low-pass characteristics of some conedriven monkey ganglion cells at least under low-to-moderate mean luminances (Purpura et al., 1990). On the other hand, voltage responses from cat horizontal cells (in direct contact with cones) already show some attenuation of low temporal frequencies (Lankheet et al., 1992). For the sake of simplicity, we lump all differentiation into one post-receptor stage.

With respect to "direct" recordings of the cone impulse response, it is a general problem whether "dim-flash" responses are really small enough to represent the waveform of responses that underlie threshold detection. For example, dark-adapted human foveal vision can detect flashes of light that deliver much less than 1 photon per cone on average, but the "dim-flash" responses shown by Schnapf et al. (1990) (1-2 pA) are 50 times larger than the estimated single-photon response $(30 \mathrm{fA})$. This may be critical, because in several species response overshoots have been found to increase with increasing flash intensities (Baylor et al., 1974; Perry \& McNaughton, 1991; Schneeweis \& Schnapf, 1995; Donner et al. unpublished observations).

\section{Response phase}

The phase shift between the stimulus and the center response (i.e. excluding surround effects) has been analyzed in terms of three main physiological determinants. Phase lags are contributed by the low-pass filtered time course of the photoreceptor signals (phase lag $\delta_{h}$ ) and by a fixed transmission delay $d_{c}$ (phase lag $\delta_{c}$ ). A phase advance is contributed by signal differentiation in the retina. The latter factor is important particularly in light-adapted states and at low frequencies; it becomes negligible above $10 \mathrm{~Hz}$.

If phase lags were entirely dominated by the constant delay $d_{c}$, a plot of phase vs. temporal frequency on linear scales would be a straight line with slope (cycles $\mathrm{Hz}^{-1}$ ) equal to $d_{c}(\mathrm{~s})$. This situation is approximated at very high frequencies. However, the modelled center phase relation on linear coordinates is a gently curved line whose slope decreases with increasing temporal frequency. At low frequencies, assuming Poisson kinetics, $\delta_{h} / \delta_{c}$ is approximately $1 / D_{c}=t_{i} / d_{c}$ [cf. eqn. (27)], and given the values of $t_{i}$ and $d_{c}$ in Table 1 it is evident that the lag due to photoreceptors is then the dominating component. Thus, for example, a major part of the illumination-dependent phase changes quantified for 2- $\mathrm{Hz}$ stimulation by Troy et al. (1993) would be due to the acceleration of photoreceptor responses.

In the resultant response, surround antagonism contributes a further phase shift $\left(\delta_{a}\right)$. The contribution is largest at low $F$ and $F_{s}$, where it appears as an advance (see Fig. 4D), reversing into a small lag at $F=\pi / 2$. Thus, the surround contribution tends to "straighten out" the relation between phase and temporal frequency. When a recorded phase function is approximately linear, this need not reflect a simple delay, but may arise as a balance of several contributions.

\section{Composite nature of the transmission delay}

One empirical justification for using a pure transmission delay $\left(d_{c}\right)$ is that GC response latencies under flash or step stimulation can be broken down into a (photoreceptor) part that decreases monotonically with stimulus intensity, plus a constant part that is independent of intensity (Donner, 1989; Donner et al., 1995). Thus, $d_{c}$ is a useful parameter for relating peri- odic and aperiodic data. At a higher level of resolution, $d_{c}$ could certainly be decomposed into both pure delays (such as signal propagation along axons) and filter stages associated, for example, with electrical properties of synaptic membranes. The basic reason why a simple delay works so well is probably that the time constants of the latter processes are small compared with those that limit the speed of photoreceptor responses. The most serious objection against a pure delay is that transmission from cones to on-bipolars is mediated by metabotropic glutamate receptors and a transduction cascade, which could increase the apparent number of stages in the Poisson response here ascribed to photoreceptors. This would, for example, make the high-frequency roll-off of the temporal modulation transfer function steeper than predicted from photoreceptor responses (cf. Fig. 4C).

Photoreceptors themselves have been modelled with "effective delays" (Lamb \& Pugh, 1992), and our way of extracting "transmission" delays from GC latencies would at least partly include these in $d_{c}$ (Table 1). Mathematically, it is only a matter of taste whether they are included in $d_{c}$ or in $f(t)$. The total center phase lag $\delta_{c}$ and the responsivity will in both cases be the same. Physiologically, the distinction is important, but analysis of the earliest rise of the human $a$-wave suggests that the photoreceptor delay is no more than 2-3 ms for mammalian rods and less than $1 \mathrm{~ms}$ for cones, so in any case it will be a minor component (Hood \& Birch, 1993a,b).

\section{Psychophysics}

We are of course not the first to note that, in human psychophysics, spatial modulation sensitivities may resemble relations produced by the DOG model, and temporal modulation sensitivities Fourier transforms of filter-cascade kinetics. Still, we feel that (justified) awe for the many complexities that can in principle affect psychophysical performance may obscure the fact that the relation to retinal function could in some situations be rather transparent. We have already discussed the relation of "surround" attenuation factors $P_{s}$ and $P_{t}$ to the "pathway" high-pass filters $P_{\mathrm{MTF}}$ of Rovamo et al. (1993, 1994, 1995).

The possible simplicity of spatio-temporal sensitivity in carefully controlled conditions is illustrated in Fig. 6, where Kelly's (1979) stabilized spatio-temporal response surface is juxtaposed with a DOG model simulation. The true differences are in fact even smaller than apparent, because in the region that looks most different (low spatial and high temporal frequency), Kelly's contours are extrapolations. The main confounding parameter value in the DOG fit is the low $\alpha$, corresponding to $t_{p}=44 \mathrm{~ms}$ (typical of dark-adapted cone kinetics, see Table 1). This, however, is a general problem with Kelly's data, where sensitivity to high-frequency flicker is much lower than shown by other studies at comparable illumination levels ( $300 \mathrm{td}$ ) (see e.g. Roufs, 1972).

Psychophysical detection of course has several advantages over detection by single GCs: averaging across many cells (improving the signal/noise ratio of an extended target) and taking recourse to those cells out of a large population that perform best in a given situation (characteristics that, moreover, strongly depend on eccentricity). Thus it is in a way amazing, particularly as regards the spatial domain, how well the psychophysical data can be mimicked by the DOG model for a single cell. One possible explanation for this may be as follows: 


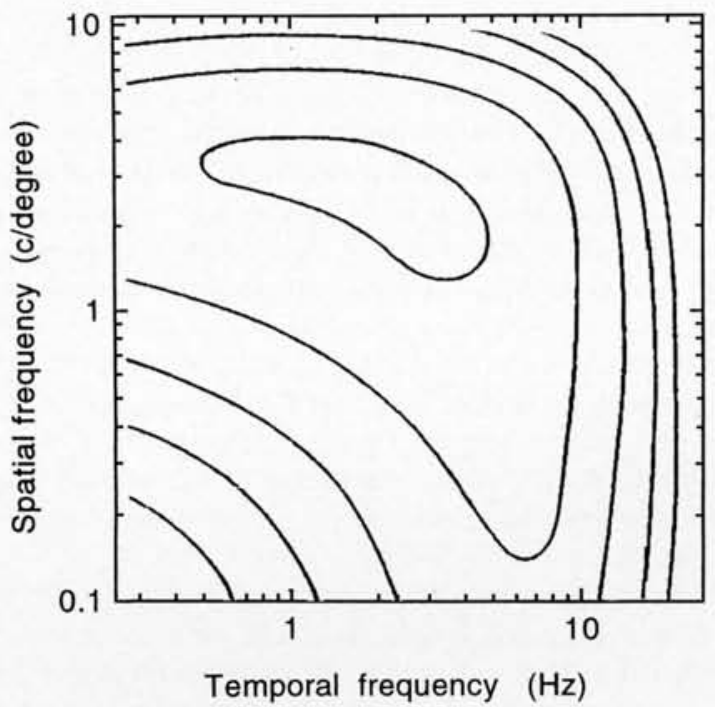

A



Temporal frequency $(\mathrm{Hz})$

B

Fig. 6. Simulation of psychophysical spatio-temporal sensitivities with the DOG model. In both (A) and (B), spatio-temporal sensitivity surfaces are displayed by isosensitivity contours at $0.3 \log _{10}$ unit intervals; the first (innermost) contour indicates $99.5 \%$ of peak sensitivity. (A) "Stabilized" spatio-temporal threshold surface (i.e. effects of eye movements eliminated) modified from Kelly (1979). (B) Model simulation. Temporal parameters: Poisson response with $n=7, \alpha=135 \mathrm{~s}^{-1}, 70 \%$ high-pass filtered with time constant $400 \mathrm{~ms}$, and surround delay $d=$ 9 ms. Spatial parameters: RFC radius $r_{0 c}=0.047 \mathrm{deg}, \mathrm{RFS} / \mathrm{RFC}$ radius ratio $R=3$, and strength ratio $K=0.95$.

Psychophysical sensitivities are expected to be equal to or better than single-cell sensitivities in absolute terms. If the improvement factor would be the same at all spatial (and, of course, temporal) frequencies, the sensitivity surface would retain its shape. Psychophysical threshold detection is known to use at least two types of averaging mechanisms that will affect detection of low and high spatial frequencies differently. Simple spatial summation will favor detection of low spatial frequencies, because wider grating bars allow more extensive summation. The integration of spatial detail, on the other hand, might favor detection of high spatial frequencies, because a stim- ulus of limited size will then encompass a larger number of cycles. Integration of cycles over stimulus area is effective up to a limit defined by a certain number of square cycles (Rovamo et al., 1993). With fixed stimulus area, it may be that only high spatial frequencies are accommodated up to the full limit. Thus it is in principle possible that, in some conditions, psychophysical sensitivities at all spatial frequencies are improved by similar factors compared with single-cell data, effecting only a general elevation of the modulation sensitivity surface without much distortion.

\section{Acknowledgments}

This work was supported by the Academy of Finland (Grants 1011872 and 1011881). We wish to thank Dr. Laura Frishman for providing us with details on several important points related to the data in Frishman et al. (1987). Thanks are due also to Dr. Ari Koskelainen for valuable discussions and help with the figures.

\section{References}

BARLOW, H.B. (1953). Summation and inhibition in the frog's retina. Journal of Physiology 119, 69-88.

BARON, W.S. \& BOYNTON, R.M. (1975). Response of primate cones to sinusoidally flickering homochromatic stimuli. Journal of Physiology 246, 311-331.

BAylor, D.A. \& Fettiplace, R. (1977a). Transmission from photoreceptors to ganglion cells in turtle retina. Journal of Physiology 271, 391-424.

BAylor, D.A. \& Fettiplace, R. (1977b). Kinetics of synaptic transfer from receptors to ganglion cells in turtle retina. Journal of Physiology 271, 425-448.

BAYLOR, D.A. \& FetTIPLACE, R. (1979). Synaptic drive and impulse generation in ganglion cells of turtle retina. Journal of Physiology 288, 107-127.

BAYLOR, D.A. \& FUORTES, M.G.F. (1970). Electrical responses of single cones in the retina of the turtle. Journal of Physiology 207, 77-92.

BAYLOR, D.A., Hodgkin, A.L. \& LAMB, T.D. (1974). The electrical response of turtle cones to flashes and steps of light. Journal of Physiology 242, 685-727.

BAYLOR, D.A., LAMB, T.D. \& YAU, K.-W. (1979). The membrane current of single rod outer segments. Journal of Physiology 288, 589-611.

BAYLOR, D.A., NunN, B.J. \& Schnapf, J.L. (1984). The photocurrent, noise and spectral sensitivity of rods of the monkey Macaca fascicularis. Journal of Physiology 357, 575-607.

Bolz, J., Rosner, G. \& WÄssLe, H. (1982). Response latency of brisksustained $(\mathrm{X})$ and brisk-transient $(\mathrm{Y})$ cells in the cat retina. Journal of Physiology 328, 171-190.

CHEN, E.P. \& FreEMAN, A.W. (1989). A model for spatiotemporal frequency responses in the $\mathrm{X}$ cell pathway of the cat's retina. Vision Research 29, 271-291.

Creutzfeldt, O.D., Sakmann, B., Scheich, H. \& Korn, A. (1970). Sensitivity distribution and spatial summation within receptive-field centre of retinal on-centre ganglion cells and transfer function of the retina. Journal of Neurophysiology 33, 654-671.

DALY, S.J. \& NoRMANN, R.A. (1985). Temporal information processing in cones: Effect of light adaptation on temporal summation and modulation. Vision Research 25, 1197-1206.

Dawis, S., Shapley, R., Kaplan, E. \& Tranchina, D. (1984). The receptive field organization of X-cells in the cat: Spatiotemporal coupling and asymmetry. Vision Research 24, 549-564.

DE LANGE, H. (1952). Experiments on flicker and some calculations on an electrical analogue of the foveal systems. Physica 18, 935-950.

DE LANGE, H. (1958). Research into the dynamic nature of the human fovea-cortex systems with intermittent and modulated light. I. Attenuation characteristics with white and colored light. Journal of the Optical Society of America 48, 777-784.

Derrington, A.M. \& LenNie, P. (1982). The influence of temporal frequency and adaptation level on receptive field organization of retinal ganglion cells in the cat. Journal of Physiology 333, 343-366.

Detwiler, P.B., Hodgkin, A.L. \& McNaughton, P.A. (1980). Tem- 
poral and spatial characteristics of the voltage response of rods in the retina of the snapping turtle. Journal of Physiology 300, 213-250.

DONNER, K. (1981). How the latencies of excitation and inhibition determine ganglion cell thresholds and discharge patterns in the frog. Vision Research 21, 1689-1692.

DONNER, K. (1989). Visual latency and brightness: An interpretation based on the responses of rods and ganglion cells in the frog retina. Visual Neuroscience 3, 39-51.

Donner, K., Duupsund, K., Reuter, T. \& VÄIsÄnen, I. (1991). Adaptation to light fluctuations in the frog retina. Neuroscience Research (Suppl.) 15, S175-S184.

Donner, K., Koskelainen, A., Djupsund, K. \& Hemilä, S. (1995). Changes in retinal time scale under background light: Observations on rods and ganglion cells in the frog retina. Vision Research 35, 2255-2266.

ENRoth-Cugell, C. \& LenNIE, P. (1975). The control of retinal ganglion cell discharge by receptive field surrounds. Journal of Physiology 247, 551-578.

ENRoth-Cugell, C. \& Robson, J.G. (1966). The contrast sensitivity of retinal ganglion cells of the cat. Journal of Physiology 187, $517-552$.

EnRoth-Cugell, C. \& RoBson, J.G. (1984). Functional characteristics and diversity of cat retinal ganglion cells. Basic characteristics and quantitative description. Investigative Ophthalmology and Visual Science 25, 250-267.

Enroth-Cugell, C., Robson, J.G., Schweitzer-Tong, D.E. \& WATson, A.B. (1983). Spatio-temporal interactions in cat retinal ganglion cells showing linear spatial summation. Journal of Physiology 341, 279-307.

ENRoth-Cugell, C. \& Shapley, R.M. (1973). Flux, not retinal illumination, is what cat retinal ganglion cells really care about. Journal of Physiology 233, 311-326.

Frishman, L.J., Freeman, A.W., Troy, J.B., Schweitzer-Tong, D.E. \& ENROTH-Cugeli, C. (1987). Spatiotemporal frequency responses of cat retinal ganglion cells. Journal of General Physiology 89, 599-628.

Frishman, L.J. \& Linsenmeier, R.A. (1982). Effects of picrotoxin and strychnine on nonlinear responses of Y-type cat retinal ganglion cells. Journal of Physiology 324, 347-363.

Fuortes, M.G.F. \& HodgKin, A.L. (1964). Changes in time scale and sensitivity in the ommatidia of Limulus. Journal of Physiology 156, 179-192.

GourAs, P. \& LinK, K. (1966). Rod and cone interaction in dark-adapted monkey ganglion cells. Journal of Physiology 184, 499-510.

GrahaM, N. \& Hood, D.C. (1992). Modeling the dynamics of light adaptation: The merging of two traditions. Vision Research 32, 1373-1393.

GrüSSER, O.J. \& GRÜSSER-CORNEHLS, U. (1973). Neuronal mechanisms of visual movement perception and some psychophysical and behavioral correlations. In Handbook of Sensory Physiology VII/3A. Central Processing of Visual Information, ed. JUNG, R., pp. 333-429. Berlin, Germany: Springer.

Hochstein, S. \& Shapley, R.M. (1976a). Quantitative analysis of retinal ganglion cell classifications. Journal of Physiology 262, 237-264.

Hochstein, S. \& SHAPLeY, R.M. (1976b). Linear and nonlinear spatial subunits in Y cat retinal ganglion cells. Journal of Physiology 262, 265-284.

HooD, D.C. \& BIRCH, D.G. (1993a). Light adaptation of human rod receptors: The leading edge of the human a-wave and models of rod receptor activity. Vision Research 33, 1605-1618.

HooD, D.C. \& BIRCH, D.G. (1993b). Human cone receptor activity: The leading edge of the $a$-wave and models of receptor activity. Visual Neuroscience 10, 857-871.

KeLLY, D.H. (1979). Motion and vision. II. Stabilized spatio-temporal threshold surface. Journal of the Optical Society of America 69, 1340-1349.

Kolв, H. (1994). The architecture of functional neural circuits in the vertebrate retina. Investigative Ophthalmology and Visual Science 35, 2385-2404.

KRAFT, T.W., SChNeEWEIS, D.M. \& SchnapF, J.L. (1993). Visual transduction in human rod photoreceptors. Journal of Physiology 464, $747-765$.

KUFFLER, S.W. (1953). Discharge patterns and functional organization of mammalian retina. Journal of Neurophysiology 16, 37-68.

LAMB, T.D. \& PUGH, E.N., JR. (1992). A quantitative account of the activation steps involved in phototransduction in amphibian photoreceptors. Journal of Physiology 449, 719-758.
Lankheet, M.J.M., Prickaerts, J.H.H.J. \& van de Grind, W.A. (1992). Responses of cat horizontal cells to sinusoidal gratings. Vision Research 32, 997-1008.

Linsenmeitr, R.A., Frishman, L.J., Jakiela, H.G. \& Enroth-Cugell, C. (1982). Receptive field properties of X and Y cells in the cat retina derived from contrast sensitivity measurements. Vision Research 22, 1173-1183.

MILLER, R.F. \& DACHEUX, R.F. (1976). Synaptic organization and ionic basis of on and off channels in mudpuppy retina. III. A model of ganglion cell receptive field organization based on chloride-free experiments. Journal of General Physiology 67, 679-690.

NYE, P.W. \& NAKA, K.-I. (1971). The dynamics of inhibitory interaction in a frog receptive field: A paradigm of paracontrast. Vision Research 11, 377-392.

Peichl, L. \& Wässle, H. (1979). Size, scatter and coverage of ganglion cell receptive field centres in the cat retina. Journal of Physiology 291, 117-141.

PeIchl, L. \& W ÄSSLE, H. (1983). The structural correlate of the receptive field centre of Ó ganglion cells in the cat retina. Journal of Physiology 341, 309-324.

PerRy, R.J. \& MCNaughton, P.A. (1991). Response properties of cones from the retina of the tiger salamander. Journal of Physiology 433, 561-587.

Purpura, K., Tranchina, D., Kaplan, E. \& Shapley, R.M. (1990). Light adaptation in the primate retina: Analysis of changes in gain and dynamics of monkey retinal ganglion cells. Visual Neuroscience 4, 75-93.

RODIECK, R.W. (1965). Quantitative analysis of cat retinal ganglion cell response to visual stimuli. Vision Research 5, 583-601.

RODIECK, R.W. \& STONE, J. (1965a). Response of cat retinal ganglion cells to moving visual patterns. Journal of Neurophysiology 28, 819-832.

Rodieck, R.W. \& STONE, J. (1965 b). Analysis of receptive fields of cat retinal ganglion cells. Journal of Neurophysiology 28, 833-849.

RouFs, J.A.J. (1972). Dynamic properties of vision. - I. Experimental relation between flicker and flash thresholds. Vision Research $\mathbf{1 2}$, 261-278.

Rovamo, J., Luntinen, O. \& NÄSÄNEN, R. (1993). Modelling the dependence of contrast sensitivity on grating area and spatial frequency. Vision Research 33, 2773-2788.

Rovamo, J., Mustonen, J. \& NÄSÄNEN, R. (1994). Modelling contrast sensitivity as a function of retinal illuminance and grating area. Vision Research 34, 1301-1314.

Rovamo, J., Raninen, A., Lukkarinen, S. \& Donner, K. (1995). Flicker sensitivity as a function of the spectral density of external white temporal noise. Vision Research (in press).

SCHNAPF, J.L., NunN, B.J., MEISTER, M. \& BAYLOR, D.A. (1990). Visual transduction in cones of the monkey Macaca fascicularis. Journal of Physiology 427, 681-713.

Schneeweis, D.M. \& Schnapf, J.L. (1995). Photovoltage of rods and cones in the macaque retina. Science 268, 1053-1056.

Shapley, R., Kaplan, E. \& Purpura, K. (1993). Contrast sensitivity and light adaptation in photoreceptors or in the retinal network. In Contrast Sensitivity, ed. SHAPLEY, R. \& LAM, D.M., pp. 103116. Cambridge, Massachusetts: The MIT Press.

TAMURA, T., NAKATANI, K. \& YAU, K.-W. (1989). Light adaptation in cat retinal rods. Science $245,755-758$.

TAMURA, T., NaKatani, K. \& Yau, K.-W. (1991). Calcium feedback and sensitivity regulation in primate rods. Journal of General Physiology 98, 95-130.

Troy, J.B. (1993). Modeling the receptive fields of mammalian retinal ganglion cells. In Contrast Sensitivity, ed. SHAPLEY, R. \& LAM, D.M., pp. 85-102. Cambridge, Massachusetts: The MIT Press.

TroY, J.B., OH, J.K. \& Enroth-Cugell, C. (1993). Effect of ambient illumination on the spatial properties of the center and surround of Y-cell receptive fields. Visual Neuroscience 10, 753-764.

VICTOR, J.D. (1987). The dynamics of the cat retinal X cell centre. Journal of Physiology 386, 219-246.

WÄsSLE, H. \& CREUTZFELDT, O.D. (1973). Spatial resolution in visual system: A theoretical and experimental study on single units in the cat's lateral geniculate body. Journal of Neurophysiology 36, 13-27.

Wässle, H., Peichl, L. \& Boycott, B.B. (1981). Dendritic territories of cat ganglion cells. Nature 292, 344-345.

WATSON, A.B. (1986). Temporal sensitivity. In: Handbook of perception and human performance, ed. Boff, K.R., KaUfman, L. \& Thomas, J.P., pp. 6.1-6.43. New York: John Wiley and Sons. 
Werblin, F.S. \& Copenhagen, D.R. (1974). Control of retinal sensitivity. III. Lateral interactions at the inner plexiform layer. Journal of General Physiology 63, 88-110.

WINTERS, R.W. \& HAMASAKI, D.J. (1976). Temporal interactions of peripheral inhibition of sustained and transient ganglion cells in cat retina. Vision Research 16, 37-45.

YAU, K.-W. (1994). Phototransduction mechanism in retinal rods and cones. Investigative Ophthalmology and Visual Science 35, 9-32.

\section{Appendix 1: The spatial factor $G\left(F_{s}\right)$}

In the case of Gaussian RF

$$
z=e^{-r^{2} / r_{0}^{2}}=e^{-x^{2} / r_{0}^{2}} e^{-y^{2} / r_{0}^{2}}
$$

the spatial function $G$ is according to eqn. (12)

$$
\begin{aligned}
G & =A_{i}^{-1} \int_{-\infty}^{\infty} \int_{-\infty}^{\infty} \cos (k x) e^{-x^{2} / r_{0}^{2}} e^{-y^{2} / r_{0}^{2}} \mathrm{~d} x \mathrm{~d} y \\
& =A_{i}^{-1}\left[\int_{-\infty}^{\infty} \cos (k x) e^{-x^{2} / r_{0}^{2}} \mathrm{~d} x\right]\left[\int_{-\infty}^{\infty} e^{-y^{2} / r_{0}^{2}} \mathrm{~d} y\right]
\end{aligned}
$$

The value of the second integral is $\sqrt{\pi r_{0}}$, and the value of the first integral is $\sqrt{\pi r_{0}} \exp \left(-k^{2} r_{0}^{2} / 4\right)$. Taking into account that $A_{i}=\pi r_{0}^{2}$, the spatial function thus reduces to

$$
G=e^{-k^{2} r_{0}^{2} / 4}=e^{-\left(\pi r_{0} f_{s}\right)^{2}}=e^{-\pi^{2} F_{s}^{2}}
$$

According to this equation the function $G$ depends only on the product $F_{s}=r_{0} f_{s}: G=G\left(F_{s}\right)$.

\section{Appendix 2: The temporal factor $H(F)$}

According to eqns. (20)-(22), the factor $H$ is

$$
\begin{aligned}
& H=h_{0} / t_{i}=t_{i}^{-1} \sqrt{h_{1}^{2}+h_{2}^{2}} \\
& h_{1}=\int_{0}^{\infty} f(\tau) \cos (\omega \tau) \mathrm{d} \tau \\
& h_{2}=\int_{0}^{\infty} f(\tau) \sin (\omega \tau) \mathrm{d} \tau
\end{aligned}
$$

Substituting the Poisson response, eqn. (3), for $f(\tau)$ leads to

$$
h_{1}=C_{P} \int_{0}^{\infty} \tau^{n-1} e^{-\alpha \tau} \cos (\omega \tau) \mathrm{d} \tau
$$

$$
h_{2}=C_{P} \int_{0}^{\infty} \tau^{n-1} e^{-\alpha \tau} \sin (\omega \tau) \mathrm{d} \tau
$$

The analytic expressions for these definite integrals can be found in mathematical tables. CRC Handbook of Chemistry and Phys$i c s$ gives such expressions in complex form [eqns. (676) and (677) on p. A62 in the 67th edition]:

$$
\begin{aligned}
& h_{1}=C_{P} \frac{(n-1) !}{2\left(\alpha^{2}+\omega^{2}\right)^{n}}\left[(\alpha+i \omega)^{n}+(\alpha-i \omega)^{n}\right] \\
& h_{2}=-C_{P} i \frac{(n-1) !}{2\left(\alpha^{2}+\omega^{2}\right)^{n}}\left[(\alpha+i \omega)^{n}-(\alpha-i \omega)^{n}\right]
\end{aligned}
$$

Although these expressions are in complex form, the integrals are in fact real. Substituting

$$
\begin{gathered}
\alpha \pm i \omega \equiv r e^{ \pm i \Phi}, \quad r=\sqrt{\alpha^{2}+\omega^{2}}, \quad \tan \Phi=\omega / \alpha \\
(\alpha \pm i \omega)^{n}=r^{n} e^{ \pm i n \Phi}
\end{gathered}
$$

leads to real expressions

$$
\begin{aligned}
& h_{1}=C_{P} \frac{(n-1) !}{\left(\alpha^{2}+\omega^{2}\right)^{n / 2}} \cos (n \Phi) \\
& h_{2}=C_{P} \frac{(n-1) !}{\left(\alpha^{2}+\omega^{2}\right)^{n / 2}} \sin (n \Phi)
\end{aligned}
$$

Because $\sin ^{2}(n \Phi)+\cos ^{2}(n \Phi)=1$, the expression for $H$ thus becomes

$$
H=\frac{C_{P}}{t_{i}} \frac{(n-1) !}{\left(\alpha^{2}+\omega^{2}\right)^{n / 2}}
$$

According to eqn. (5), $C_{P} / t_{i}=\alpha^{n} /(n-1)$ ! and we obtain

$$
H=\left(1+\omega^{2} / \alpha^{2}\right)^{-n / 2}
$$

The shape parameter $N$ is according to eqn. (25)

$$
N=2 \pi /\left(\alpha t_{i}\right)
$$

Thus, $\omega / \alpha=N f t_{i}=N F$ and the final expression for $H$ is

$$
H=\left(1+N^{2} F^{2}\right)^{-n / 2}
$$

